\title{
Aspirin acetylates multiple cellular proteins in HCT-116 colon cancer cells: Identification of novel targets
}

\author{
SRINIVASAN MARIMUTHU ${ }^{1}$, RAGHAVENDER S.V. CHIVUKULA ${ }^{1}$, LLOYD F. ALFONSO ${ }^{2}$, \\ MAJID MORIDANI ${ }^{3}$, FRED K. HAGEN ${ }^{4}$ and G. JAYARAMA BHAT ${ }^{1}$
}

${ }^{1}$ Department of Biomedical Sciences, Texas Tech University Health Sciences Center, 1300 Coulter Drive, Amarillo, TX 79106; ${ }^{2} D^{\prime}$ Youville College School of Pharmacy, 320 Porter Avenue, Buffalo, NY 14201; ${ }^{3}$ Department of Pharmaceutical Sciences, Texas Tech University Health Sciences Center, 1300 Coulter Drive, Amarillo, TX 79106; ${ }^{4}$ Proteomics Center, Biochemistry and Biophysics, University of Rochester School of Medicine and Dentistry, 601 Elmwood Ave., Rochester, NY 14642, USA

Received May 2, 2011; Accepted June 10, 2011

DOI: 10.3892/ijo.2011.1113

\begin{abstract}
Epidemiological and clinical observations provide consistent evidence that regular intake of aspirin may effectively inhibit the occurrence of epithelial tumors; however, the molecular mechanisms are not completely understood. In the present study, we determined the ability of aspirin to acetylate and posttranslationally modify cellular proteins in HCT- 116 human colon cancer cells to understand the potential mechanisms by which it may exerts anti-cancer effects. Using anti-acetyl lysine antibodies, here we demonstrate that aspirin causes the acetylation of multiple proteins whose molecular weight ranged from 20 to $200 \mathrm{kDa}$. The identity of these proteins was determined, using immuno-affinity purification, mass spectrometry and immunoblotting. A total of 33 cellular proteins were potential targets of aspirin-mediated acetylation, while 16 were identified as common to both the control and aspirin-treated samples. These include enzymes of glycolytic pathway, cytoskeleton proteins, histones, ribosomal and mitochondrial proteins. The glycolytic enzymes which were identified include aldolase, glyceraldehyde-3-phosphate dehydrogenase, enolase, pyruvate kinase M2, and lactate dehydrogenase A and B chains. Immunoblotting experiment showed that aspirin also acetylated glucose-6-phosphate dehydrogenase and transketolase, both enzymes of pentose phosphate pathway involved in ribonucleotide biosynthesis. In vitro assays of these enzymes revealed that aspirin did not affect pyruvate kinase and lactate dehydrogenase activity; however, it decreased glucose 6 phosphate dehydrogenase activity. Similar results were also observed in HT-29 human colon cancer cells. Selective inhibition of glucose-6-phosphate dehydrogenase may represent an important mechanism by which aspirin may exert its anti-cancer effects through inhibition of ribonucleotide synthesis.
\end{abstract}

Correspondence to: Dr G. Jayarama Bhat, Department of Biomedical Sciences, Texas Tech University Health Sciences Center, 1300 Coulter Drive, Amarillo, TX 79106, USA

E-mail: jayarama.gunaje@ttuhsc.edu

Key words: aspirin, acetylation, metabolic enzymes, glycolytic pathway, pentose phosphate pathway, anti-cancer effect

\section{Introduction}

Aspirin (acetylsalicylic acid), which is well known for its analgesic, anti-inflammatory and cardioprotective effects, also reduces the incidence of the epithelial cancers $(1,2)$. Epidemiological studies carried over the past decade showed that regular intake of aspirin can decrease the incidence of cancer of the esophagus by 73 , stomach 62 , and colon by $63 \%$. Cancer risk reduction was also observed in other organs such as breast (39\%), lung (36\%) and prostate tissue (39\%) (3). In vitro studies have shown that aspirin is effective in inhibiting cell proliferation in a variety of cancer cell lines $(4,5)$. In addition, it has been shown that regular aspirin use after the diagnosis of colorectal cancer lowers the risk and increases the overall patient survival (6). However, the molecular mechanisms leading to its anti-cancer effects are not clearly understood.

Aspirin consists of acetyl and salicylate moieties, both of which have their own distinct targets. While the salicylate group has been implicated in the anti-inflammatory properties of aspirin via the inhibition of NF- $\mathrm{KB}$ pathway (7), the acetyl group causes the inactivation of cyclooxygenases (COX) through acetylation of serine residues $(8,9)$. Cyclooxygenases exists mainly in two forms: COX1 and COX2. Several mechanisms have been proposed to explain aspirin's chemopreventive properties. One widely accepted hypothesis is that aspirin decreases the cancer incidence by inhibiting COX2, as this enzyme is progressively over-expressed during the development of human colorectal cancers, and its disruption inhibits polyp formation in mice (10). However, other potential anti-cancer mechanisms, unrelated to COX also have been proposed $(5,11)$. These include inhibition of NF- $\mathrm{KB}(7,12)$, induction of apoptosis by activation of p38 kinases (13), and catabolism of polyamines (14).

While aspirin's ability to acetylate and inhibit COX enzyme activity is well known $(8,9)$, limited information is available as to whether it can acetylate other proteins and alter their functions $(15,16)$. We recently demonstrated in MDA-MB-231 human breast cancer cells that one of the acetylation targets of aspirin is the tumor suppressor protein, $\mathrm{p} 53$ (17). In that report, we showed that aspirin acetylated lysine 382 (K382) on p53 and induced the expression of its target genes $\mathrm{p} 21^{\mathrm{CIP1}}$ and Bax (17). Acetylation of $\mathrm{p} 53$ by aspirin was also observed in HCT-116 and HT-29 human colon cancer 
cell lines (unpublished data). In another report, we showed that aspirin acetylated multiple cellular proteins in normal liver cells (18). Based on these observations, we hypothesized that exposure of other cell types to aspirin may cause acetylation of proteins, and identification of these targets would provide insight into the novel mechanisms on its anti-cancer effects.

Since significant cancer risk reduction is observed in colon tissue of patients who regularly take aspirin, we utilized human HCT-116 colon cancer cells in this study. We determined the pattern of aspirin induced acetylation of proteins using antiacetyl lysine antibody specific for the recognition of acetylated lysine residues. The identity of the proteins was established by mass spectrometry and immunoblotting. Here, we demonstrate that aspirin exposure of cells caused acetylation of cytoskeletal proteins, histones, heatshock proteins, glycolytic pathway and pentose phosphate pathway enzymes. The enzymes identified include aldolase, glyceraldehyde 3 phosphate dehydrogenase (GAPDH), phosphoglyceromutase (PGM), enolase, pyruvate kinase isoform type M2 (PKM2), and lactate dehydrogenase (LDH-A and LDH-B), all of which are glycolytic enzymes; and glucose 6 phosphate dehydrogenase (G6PD) and transketolase, in the pentose phosphate pathway (PPP). Enzyme assays showed that despite acetylation, PKM2 and LDH enzyme activities were unaffected; in contrast, G6PD activity was inhibited with increasing concentrations of aspirin. Since G6PD regulates ribonucletide biosynthesis, its targeted inhibition by aspirin may represent an important mechanism by which it exerts its anticancer effects.

\section{Materials and methods}

Materials. Cell culture reagents were purchased from Invitrogen. Aspirin was obtained from Sigma. Anti-acetyl-lysine antibody was purchased from Immunechem Inc. Anti-pyruvate kinase M2 antibody, anti-phospho PKM2 antibody, anti-LDH-A antibody and anti- $\beta$-actin antibody were obtained from Cell Signaling Technology, Inc. Anti-enolase antibody, LDH-B antibody, antiPGM antibody, anti-G6PD antibody, anti-transketolase antibody and anti-GAPDH antibody were obtained from Santa Cruz Biotechnology. All other chemicals were either from Sigma or Fisher.

Cell culture. HCT-116 and HT-29 cells were obtained from American Type Culture Collection (ATCC). Cells were grown for 12-24 $\mathrm{h}$ before the addition of aspirin for indicated times.

Preparation of cell lysate and immunoprecipitation. For immunoblotting experiments, cells were treated with aspirin for the indicated time and washed with phosphate buffered saline (PBS). Cells were scraped in lysis buffer $(10 \mathrm{mM}$ Tris-HCl, $\mathrm{pH} 7.4$, $150 \mathrm{mM} \mathrm{NaCl}, 15 \%$ glycerol, $1 \%$ Triton-X-100, $1 \mathrm{mM}$ sodium orthovanadate, $10 \mu \mathrm{g} / \mathrm{ml}$ leupeptin, $10 \mu \mathrm{g} / \mathrm{ml}$ aprotinin, $1 \mathrm{mM}$ $\mathrm{NaF}$, and $1 \mathrm{mM}$ phenylmethylsulfonyl fluoride (PMSF). Proteins $(200 \mu \mathrm{g})$ were immunoprecipitated with anti-acetyl lysine antibody conjugated to agarose overnight and washed 3 times with lysis buffer. The proteins bound to agarose were eluted with $70 \mu \mathrm{l}$ of triethylamine buffer ( $50 \mathrm{mM}$ triethylamine, $150 \mathrm{mM} \mathrm{NaCl})$ and neutralized with $12 \mu \mathrm{l}$ of $2 \mathrm{M}$ Tris (pH 6.8). The samples were then loaded onto a polyacrylamide gel, immunoblotted with respective primary antibodies. Immunoreactive bands were visualized using enhanced chemiluminescence (ECL) Western blotting system according to the manufacturer's instructions (Pierce).

Sample preparation for mass spectrometry analysis, using $L C-M S / M S$. For mass spectrometry studies, $200 \mu \mathrm{g}$ of cell lysates was immunoprecipitated using anti-acetyl lysine antibody conjugated agarose as described above. The immunoprecipitation (IP) resin was washed initially twice with lysis buffer, then once with detergent-free lysis buffer, and finally a last wash with $50 \mathrm{mM}$ ammonium bicarbonate. The bound proteins were eluted, reduced, alkylated, and digested with either trypsin, or chymotrypsin using the methods described below. The peptide digests were subjected to LC-MS/MS analysis to identify peptide sequence matches.

Proteolytic digestion. Because total protein staining with Coomassie blue dye did not detect protein in the standard immunoprecipitate, the sample was scaled up, by conducting 20 identical immunoprecipitations. Proteins bound to anti-acetyllysine antibody resin were eluted at low $\mathrm{pH}$ elution, using an acidic volatile buffer. For the 20 vials, one $150 \mu 1$ volume of a volatile acid buffer (VAB: $500 \mathrm{mM}$ acetic acid, $20 \mathrm{mM}$ ammonium acetate, $\mathrm{pH} 3.5$ ) was added to the first tube and then sequentially transferred from one tube to the next, to elute the bound proteins from all 20 tubes. The eluant from the 20th tube was transferred into a LoBind tube containing $450 \mu 1$ of $50 \mathrm{mM}$ ammonium bicarbonate, to immediately neutralize the sample. A total of three acid treatments were performed and combined into the same tube. The sample was lyophilized and resuspended in $20 \mu 1$ of $50 \mathrm{mM}$ ammonium bicarbonate. To digest the proteins, $188 \mathrm{ng}$ trypsin was added for an overnight digestion at $37^{\circ} \mathrm{C}$. The sample was reduced with $2 \mathrm{mM}$ dithiothreitol (DTT) for $30 \mathrm{~min}$, with shaking at $60^{\circ} \mathrm{C}$, and alkylated with $10 \mathrm{mM}$ iodoacetamide (IAA) for $30 \mathrm{~min}$ in the dark at room temperature (RT). Finally, the alkylation reaction was quenched with $10 \mathrm{mM}$ cysteine for 30 min at RT in the dark. A second aliquot of trypsin was added for $3 \mathrm{~h}$ to completely digest the sample. After digestion was complete, samples were lyophilized in a speedvac and resuspended in 5\% acetonitrile, $0.05 \%$ formic acid and immediately loaded on a reverse phase nano-spray tip for LC-MS/MS analysis.

Mass spectrometry, using LC-MS/MS. Peptide digest (15\%) was loaded on a Magic C18 AQ (Michrom) nanospray tip, packed to $5 \mathrm{~cm}$. This tip is loaded, using a pressure bomb, and washed after installation on the HPLC of a Thermo LTQ mass spectrometer with $5 \%$ methanol, $0.1 \%$ formic acid, for 10 min with a flow rate of $600 \mathrm{nl} / \mathrm{min}$ (about 10 column volumes $=6.6 \mu \mathrm{l}$ ). The LTQ ion trap mass spectrometer was equipped with nano-electrospray ionization source. The peptides were eluted and analyzed in an LC-MS/MS run, using a 5-15\% methanol gradient $>2.5 \mathrm{~min}$, followed by a $15-60 \%$ methanol gradient for $67 \mathrm{~min}$, a $60 \%$ methanol isocratic step of 4 min, ending with a 3-min $95 \%$ methanol step, with all solvents containing $0.1 \%$ formic acid. A full MS survey scan is performed every $3 \mathrm{sec}$ and the top 7 peaks were selected to collect MS/MS fragmentation spectrum, which were acquired in the data-dependent mode. The MS and fragmentation spectrum data was used in a Mascot search of the whole human proteome, to identify peptides derived from the proteins bound to the anti-acetylated lysine antibody column. Mascot search 
A

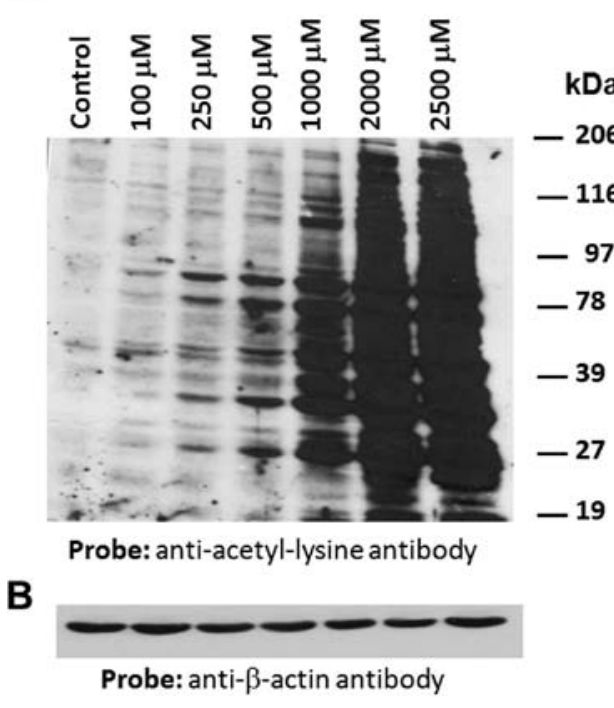

C

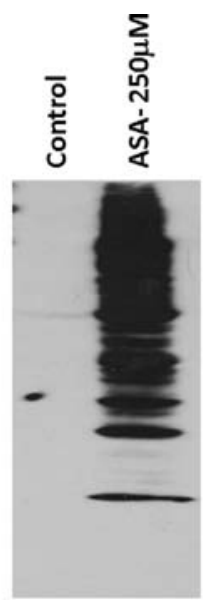

IP: rabbit polyclonal-anti-acetyl-lysine antibody Probe: mouse monoclonal-antiacetyl-lysine antibody

Figure 1. Aspirin acetylates multiple proteins in HCT-116 cells. (A) Concentration dependent protein acetylation by aspirin. Cells were grown in culture medium, left untreated or treated for $12 \mathrm{~h}$ with different concentrations of aspirin as indicated. Proteins were run on an 8\% SDS-PAGE and immunoblotted with anti-acetyl lysine antibody. Protein bands were detected using enhanced chemiluminiscence (ECL). (B) The blot in (A) was stripped and reprobed with anti- $\beta$-actin antibody (loading control). (C) Immunoprecipitation of acetylated proteins by anti-acetyl lysine antibody. Cells were left untreated or treated with aspirin ( $250 \mu \mathrm{M})$ for $12 \mathrm{~h}$, lysates prepared, $200 \mu \mathrm{g}$ was immuprecipitated with rabbit anti-acetyl lysine antibody agarose conjugate. Protein was eluted from the resins and immunoblotted using mouse monoclonal anti-acetyl lysine antibody. Protein bands were detected using ECL. These experiments were repeated three times.

parameters include: precursor and fragment ion mass tolerance were set at 1.5 and $0.8 \mathrm{Da}$, respectively, and allowed for one $\mathrm{C} 13$ incorporation. Fixed carbamodomethyl-cysteine modification, variable methionine-oxidation and variable lysine acetylation were allowed in the Mascot search against the complete human proteome (NCBI 20110407). Because acetylation blocks trypsin cleavage, three missed trypsin cleavage sites were allowed in the search. The ion score/Expected cut-off score was set for 0.1 and peptides with an ions score greater than 50 were considered positive identification, if only one peptide was identified for a given protein.

PKM2, LDH and G6PD assays. Subconflent cells were treated with aspirin at different concentration; cells lysed using the assay buffer according to the manufacturer's instructions (BioVision Inc). The enzymatic assays for PKM2, LDH, G6PD were measured in 96-well plates. In PKM2 assay, phosphoenol pyruvate and adenosine diphosphate are catalyzed by pyruvate kinase to generate pyruvate and adenosine triphosphate. The generated pyruvate is oxidized by pyruvate oxidase to produce color which is measured at $570 \mathrm{~nm}$. For LDH assay, LDH reduces NAD to NADH which then interacts with specific probe to produce a colour which is measured at $450 \mathrm{~nm}$. For the G6PD assay, glucose 6 phosphate is oxidized with the generation of a product which is utilized to convert a nearly colorless probe to an intensely colored product with an absorbance at $450 \mathrm{~nm}$.

\section{Results}

Aspirin acetylates multiple cellular proteins in human colon cancer cells. Recent reports in literature have demonstrated that acetylation of cellular proteins is a widespread phenomenon and this posttranslational modification can regulate protein functions (19-21). The array of proteins that undergo acetylation within the cells include transcription factors, histones, metabolic pathway enzymes and cytoskeletal proteins to name few. Since aspirin is known to acetylate cyclooxygenases (COX) through the non-enzymatic reaction, aminolysis (22), we hypothesized that aspirin's range of targets may also include other cellular proteins. To determine this, HCT-116 cells were treated with aspirin at different concentrations for $12 \mathrm{~h}$, lysates prepared and immunoblotted with anti-acetyl lysine antibody. Fig. 1A demonstrates that very few acetylated proteins were detected in the untreated control; however, low amounts of aspirin acetylated proteins were detected at $100 \mu \mathrm{M}$ concentration, but this increased significantly thereafter. The molecular weight of these proteins ranged from 20 to $200 \mathrm{kDa}$. Reprobing the blot with anti- $\beta$-actin antibody showed equal amounts of protein levels in all lanes (Fig. 1B; loading control). Aspirin-mediated acetylation was also observed in HT-29 cells with similar acetylation profiles (data not shown).

Identification of proteins by mass spectrometry. We then sought to determine the identity of acetylated proteins through mass spectrometry. Since the acetylation levels of proteins detected at $100 \mu \mathrm{M}$ were low, $250 \mu \mathrm{M}$ aspirin was used to treat cells to quantitatively recover the acetylated proteins in immunoprecipitation reactions. For this, cells were treated with aspirin at $250 \mu \mathrm{M}$ for $12 \mathrm{~h}$, proteins immunoprecipitated with anti-acetyl lysine antibody conjugated agarose. Multiple immunoprecipitations were carried out and pooled to ensure that enough proteins were collected for mass spectrometry. Fig. 1C demonstrates that polyclonal anti-acetyl lysine antibody successfully immunoprecipitated acetylated proteins in aspirin treated cells (lane 2). Mass spectrometry analysis identified a total of 16 proteins common between untreated control and aspirin treated samples, whereas 33 unique proteins were identified in aspirin treated samples (Tables I and II). Their reference accession numbers, function 


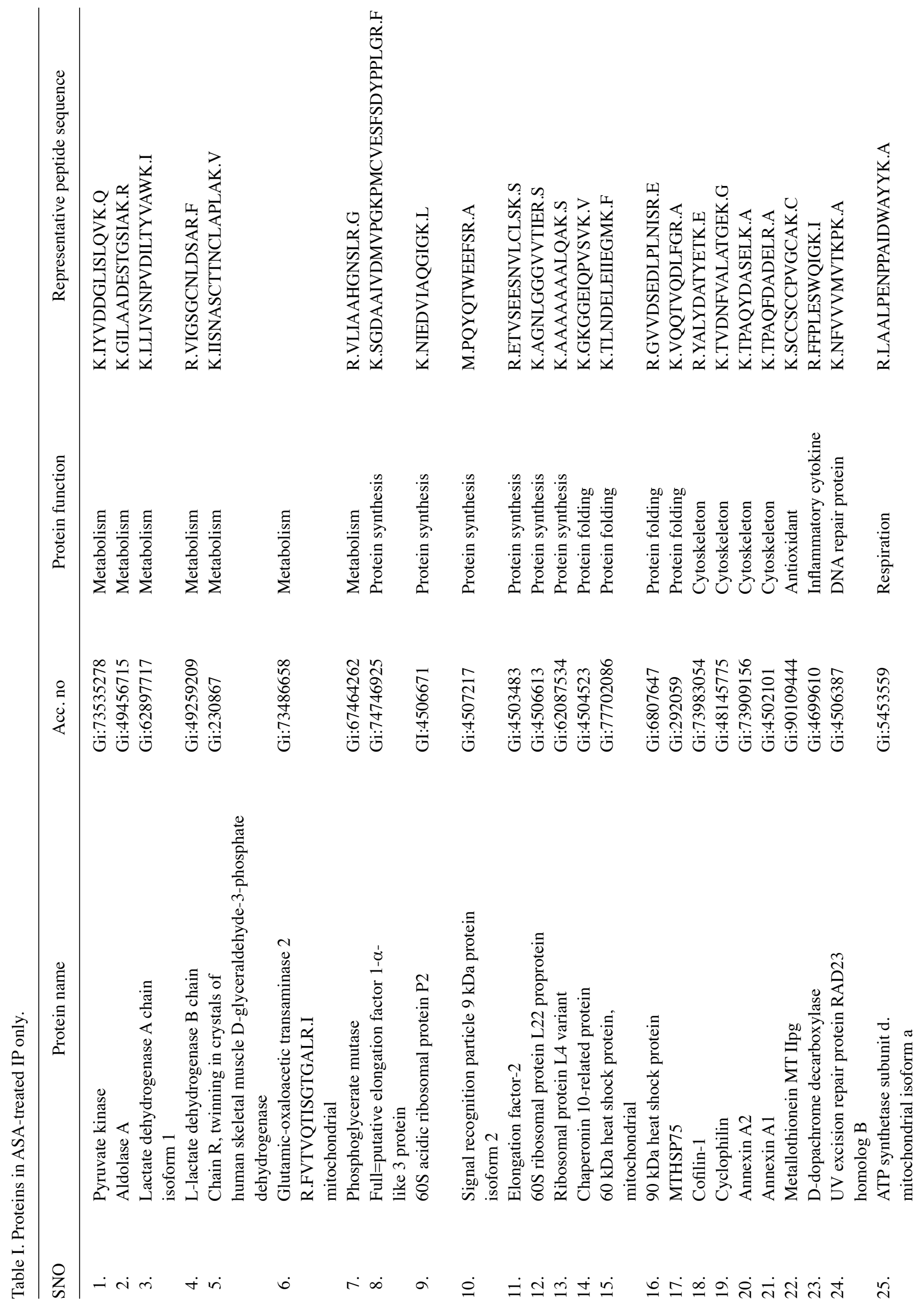




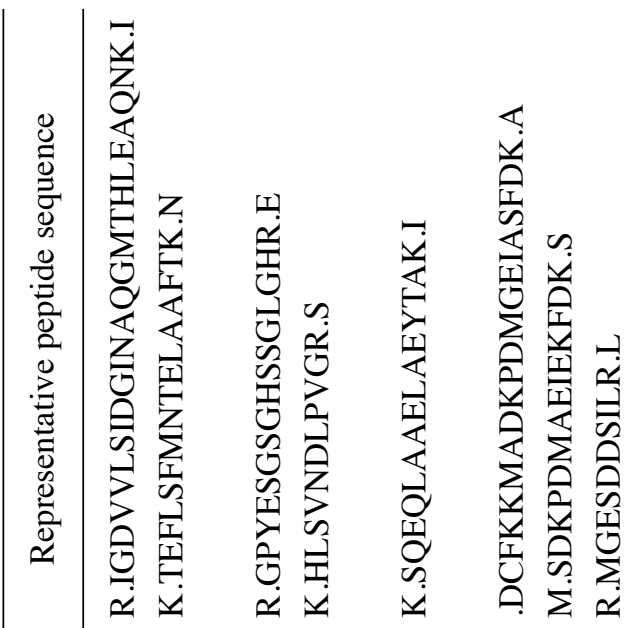

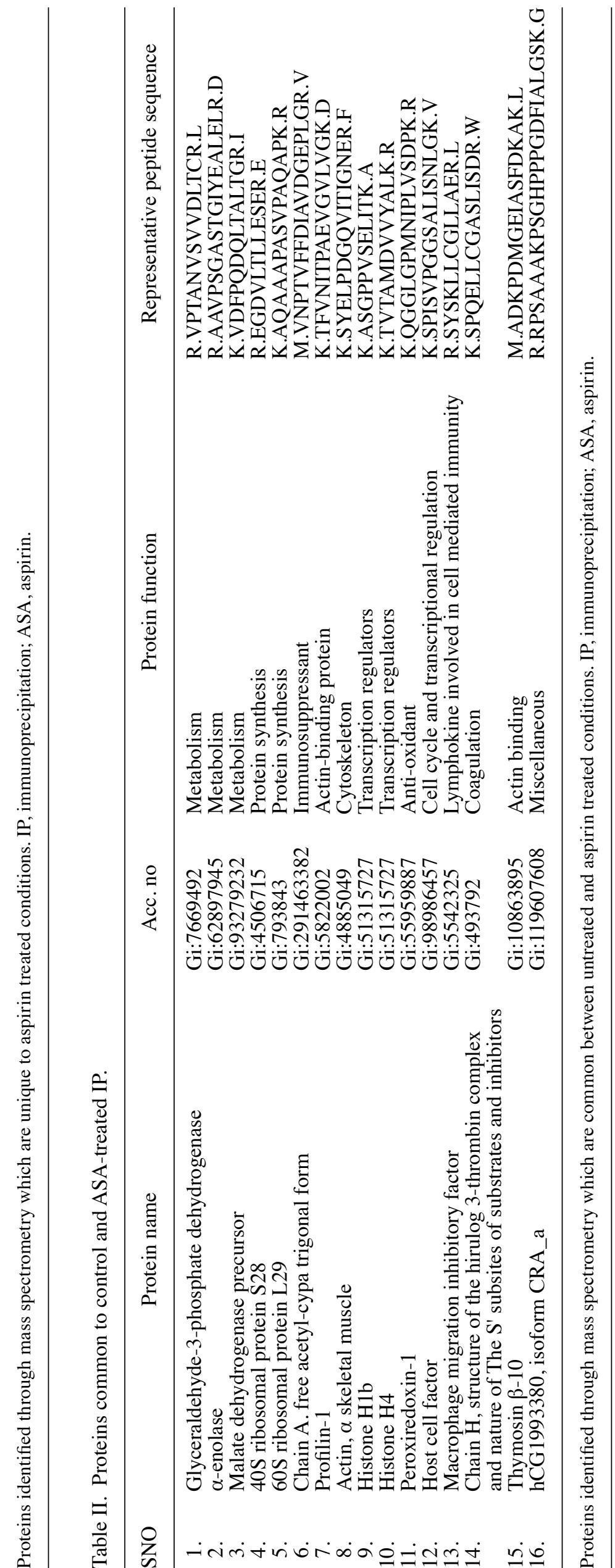

\&

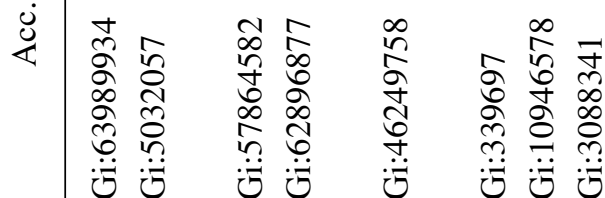

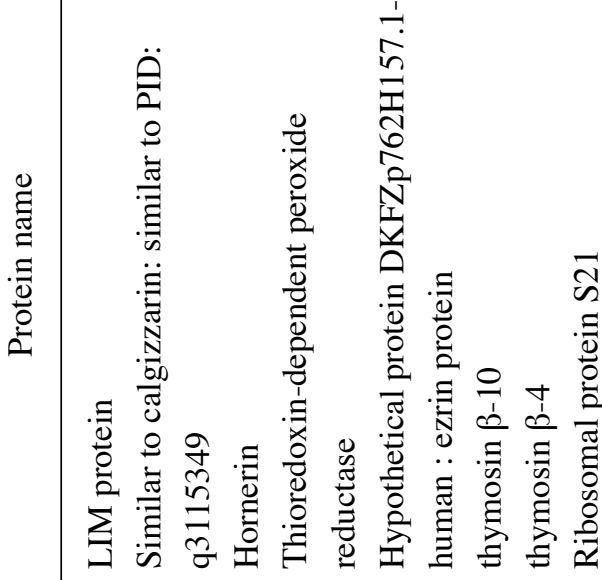

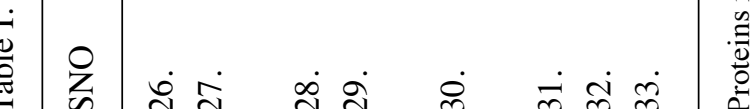

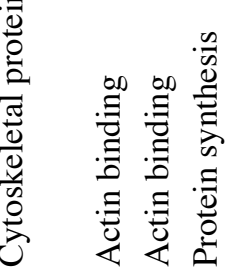

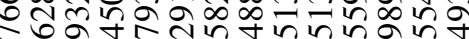

ல் 
and a representative peptide identified are shown. Some of the notable proteins in this list include metabolic pathway enzymes such as aldolase, $\alpha$-enolase, PGM, GAPDH, PKM2, LDH-A and $\mathrm{B}$ chains, glutamic-oxaloacetic transaminase 2 and malate dehydrogenase; cytoskeletal proteins such as Annexin A1 and $\mathrm{A} 2$; and DNA binding proteins such as histone $\mathrm{H} 1 \mathrm{~b}$ and histone H4. Definitive evidence for protein acetylation was achieved by identification of acetylated peptides. Mass spectrometry fragmentation spectrum analysis revealed 5 acetylated peptides from 5 aspirin-treated proteins and an additional 3 acetylated peptides from both the treated and untreated immunoprecipitated samples (Table III). The acetylated peptides map to 5 of 6 known native acetylation sites in thymolysin $\beta-10$, histone $\mathrm{H} 4$ and hCG1993380 isoform CRA, demonstrating that expected acetylation sites are successfully mapped, using LC-MS/MS method. Mass spectrometry identification of acetylated peptides specifically in the aspirin-treated sample, indicates that proteins are indeed modified by the treatment and are enriched by the immunoprecipitation protocol.

Confirmation of acetylation of glycolytic pathway enzymes by aspirin. Although mass spectrometry analysis identified many proteins as potential targets of aspirin, in this study, we focused initially on aspirin's ability to acetylate glycolytic enzymes. Because cancer cells exhibit increased rate of glycolysis and lactate production (23-25), we considered the possibility that aspirin acetylation of glycolytic enzymes might alter their function. Immunoblotting experiments were performed to further confirm the acetylation of these enzymes. In these experiments, we used aspirin at a concentration of $100 \mu \mathrm{M}$ as this concentration has been demonstrated to be physiologically achievable on administration of an oral dose (1.2 g) (26). Cell lysates were prepared from untreated and aspirin-treated cells for $12 \mathrm{~h}$, equal amounts of proteins immunoprecipitated with anti-acetyl lysine antibody agarose conjugate, proteins eluted and immunoblotted with either anti-aldolase antibody, or anti-GAPDH antibody, or anti-PGM antibody, or anti-enolase antibody, or anti-PKM2 antibody, or anti-LDH A and B antibodies. We observed that aspirin acetylated aldolase (Fig. 2A), GAPDH (Fig. 2C), PGM (Fig. 2E), enolase (Fig. 2G), PKM2 (Fig. 2I), LDH-A (Fig. 2K) and LDH-B (Fig. 2M) enzymes. Among these, enolase was constitutively acetylated and this was significantly increased following aspirin treatment (Fig. 2G). The amount of proteins between control and aspirin treated conditions remained similar suggesting that aspirin does not alter the levels of these enzymes; but causes their acetylation (Fig. 2B, D, F, H, J, L and N).

Aspirin acetylates PKM2 but does not alter phosphorylation at $Y^{105}$. PKM2 exists in two forms: the active tetrameric form; and the less active dimeric form (24). It was shown that oncoproteins such as fibroblast growth factor receptor type 1 can phosphorylate the tyrosine $105\left(\mathrm{Y}^{105}\right)$ on PKM2 and this leads to prevention of the formation of active tetrameric form and inhibition of enzyme activity (27). Since aspirin acetylates PKM2 (Fig. 2I), we determined whether this modification has any effect on the tyrosine phosphorylation at Y105 of the enzyme. For this, cells were left untreated or treated with aspirin $(100 \mu \mathrm{M})$, equal amounts of proteins immunoblotted with anti-phospho PKM2 antibody. Fig. 3A demonstrates that aspirin treatment did not alter phosphorylation at Y105

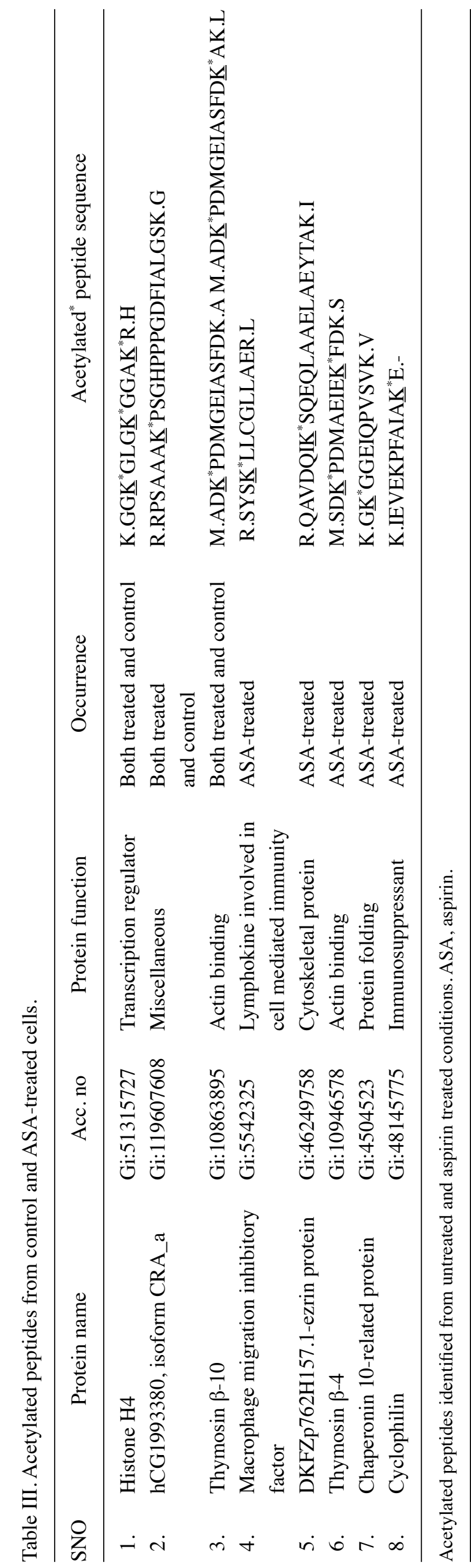




\section{A}

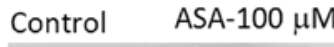
IP: anti-acetyl lysine antibody

C Probe: anti-aldolase antibody

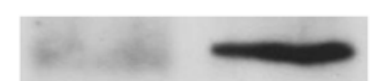

IP: anti-acetyl lysine antibody Probe: anti-GAPDH antibody

E

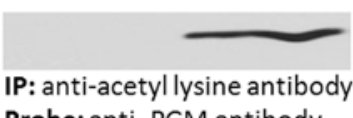

Probe: anti- PGM antibody

G

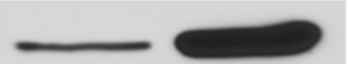

IP: anti-acetyl lysine antibody Probe: anti- enolase antibody

I

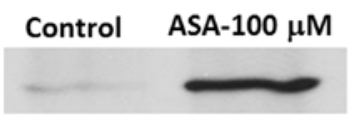

IP: anti-acetyl-lysine antibody Probe: anti-PKM2 antibody

K

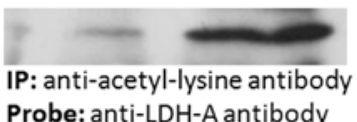

M

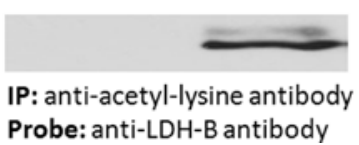

B Control ASA-100 $\mu \mathrm{M}$

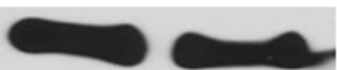

Probe: anti-aldolase antibody

D

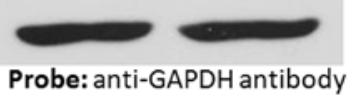

$\mathbf{F}$

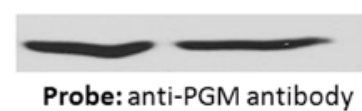

H
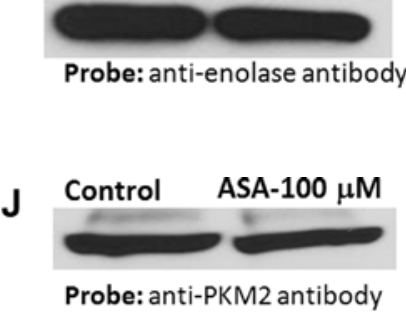

L

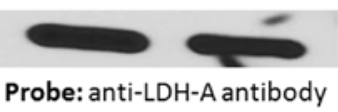

N

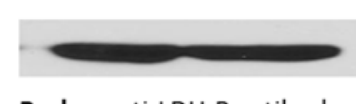

Probe: anti-LDH-B antibody

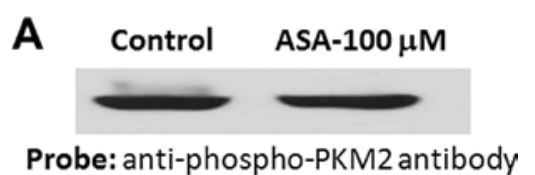

B

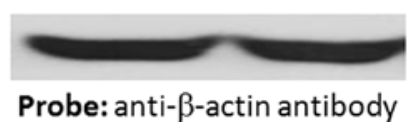

Figure 3. Aspirin does not alter PKM2 phosphorylation at $\mathrm{Y}^{105}$. (A) Cells were left untreated or treated with aspirin at $100 \mu \mathrm{M}$ for $12 \mathrm{~h}$, lysates prepared, equal amounts of proteins $(20 \mu \mathrm{g})$ loaded onto an $8 \%$ SDS-PAGE and immunoblotted with anti-phospho PKM2 antibody. (B) The blot in (A) was stripped and reprobed with anti- $\beta$-actin antibody. Protein bands were detected using ECL. These experiments were repeated four times.

A

\section{PKM2}

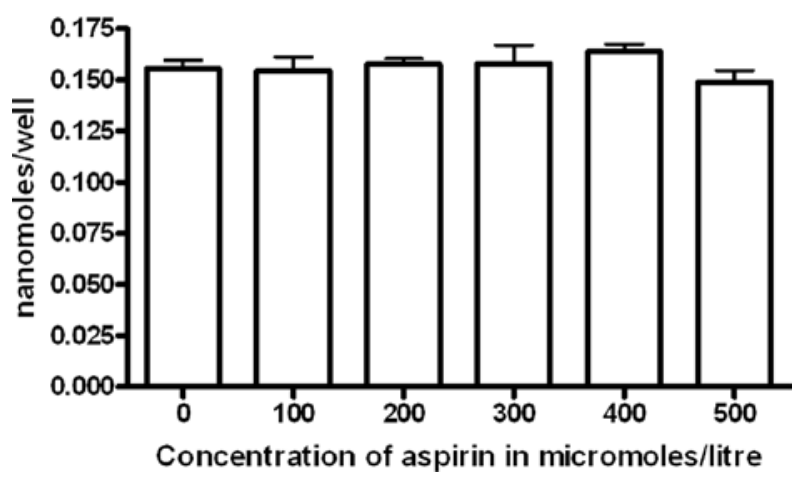

B

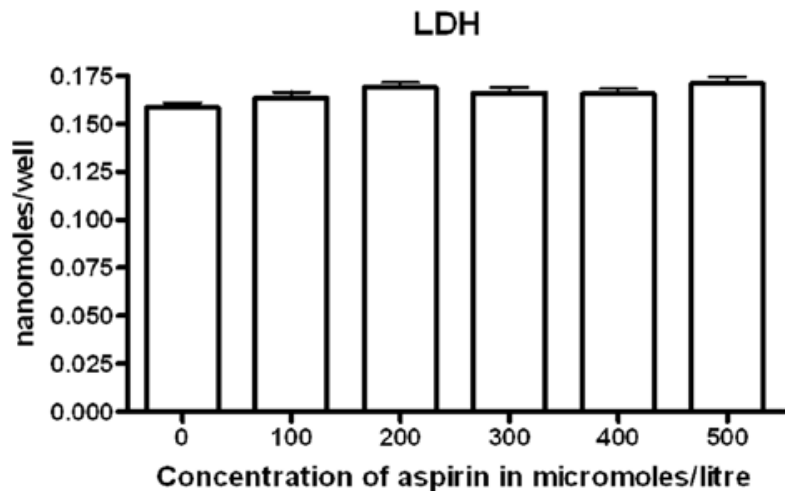

Figure 4. Aspirin does not alter the PKM2 and LDH enzyme activity. (A) Cells were seeded on $100 \mathrm{~mm}$ petridish overnight and treated with different concentrations of aspirin for $24 \mathrm{~h}$. Cells $\left(2 \times 10^{6}\right)$ were homogenized in the assay buffer using a dounce homogenizer, lysates centrifuged and supernatant collected and protein estimated. For the PKM2 activity, $1 \mu \mathrm{g}$ of the protein was used according to the protocol (Biovision Inc.). Following $30 \mathrm{~min}$ of incubation at room temperature and color development, the absorbance was measured at $570 \mathrm{~nm}$. The pyruvate that is generated was expressed in nanomoles/well. (B) The samples were prepared as in A, $0.5 \mu \mathrm{g}$ of the protein was used for the $\mathrm{LDH}$ assay according to the protocol (Biovision Inc.). Following $30 \mathrm{~min}$ of incubation at room temperature, the absorbance was measured at $450 \mathrm{~nm}$. The NADH generated was expressed in nanomoles/well. Results are means \pm SEM $(n=3)$. The data were analyzed using one-way ANOVA followed by Bonferonni's-multiple comparison test and no statistically significant change was observed across different groups.

Aspirin acetylates pentose phosphate pathway (PPP) enzymes glucose 6 phosphate dehydrogenase (G6PD) and transketolase. We considered the possibility that additional proteins may get enzyme activity over the range of concentrations tested. 
A

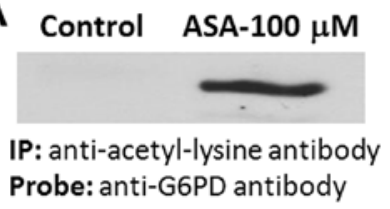

B

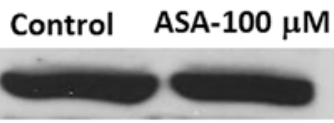

Probe: anti-G6PD antibody

\section{Control ASA-100 $\mu \mathrm{M}$

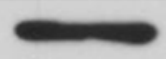 \\ IP: anti-acetyl-lysine antibody Probe: anti-transketolase antibody}

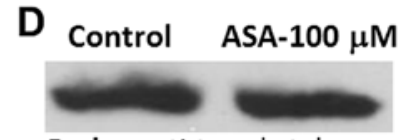

Probe: anti-transketolase antibody

Figure 5. Aspirin acetylates G6PD and transketolase. (A and C) Cells were left untreated or treated with aspirin at $100 \mu \mathrm{M}$ for $12 \mathrm{~h}$, lysates prepared, $200 \mu \mathrm{g}$ of proteins immunoprecipitated with rabbit anti-acetyl lysine antibody agarose conjugate. Protein was eluted from the resins and immunoblotted with anti-G6PD or anti-transketolase antibody. (B and D) Twenty microgram of the samples representing (A) and (C) was run on a 12\% SDS-PAGE and immunoblotted with anti-G6PD or anti-transketolase antibody. Protein bands were detected using ECL. These experiments were repeated four times.

\section{G6PD}

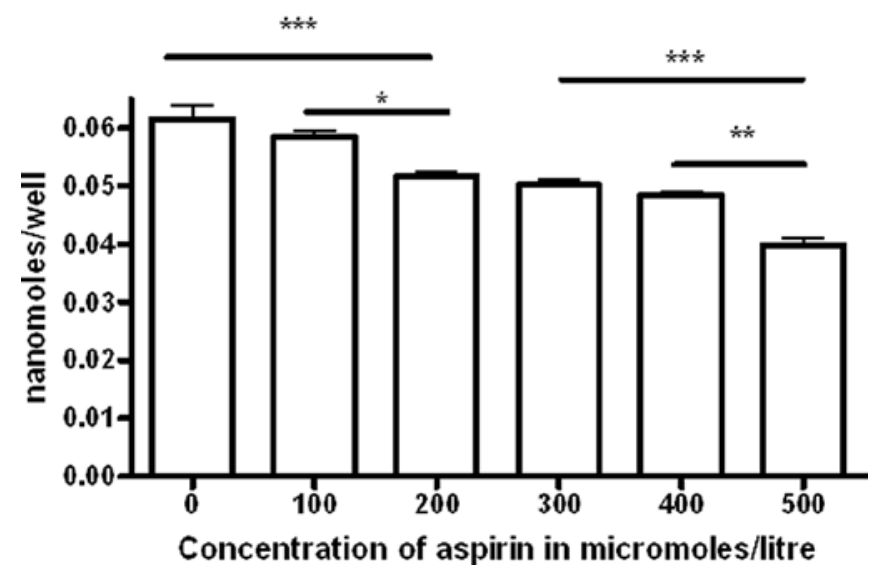

Figure 6. Aspirin inhibits G6PD enzyme activity. Cells were cultured and treated with aspirin as described in Fig. 5. One hundred $\mu \mathrm{g}$ of the protein was used for the G6PD assay according to the protocol (Biovision Inc.). The reaction mixture was incubated at $37^{\circ} \mathrm{C}$ for $30 \mathrm{~min}$, the absorbance measured at $450 \mathrm{~nm}$. The NADH generated was expressed in nanomoles/well. Results are means \pm SEM $(n=3)$. The data were analysed using one-way ANOVA followed by Bonferonni's-multiple comparison test, giving p-value between each group, p $\leq 0.001,{ }^{* *} \mathrm{p} \leq 0.01,{ }^{*} \mathrm{p} \leq 0.05$.

acetylated with aspirin, but are below the limits of detection of mass spectrometry. Moreover, some proteins may go undetected as abundant non-acetylated peptides may compete with acetylated peptides for detection (30). To explore the possibility that additional metabolic proteins such as G6PD and transketolase become acetylated, but are difficult to detect via mass spectrometry, we enriched the acetylated proteins after $100 \mu \mathrm{M}$ aspirin treatment by conducting an anti-acetyl lysine immunoprecipitation and probing with anti-G6PD and anti-transketolase antibodies. G6PD and transketolase respectively are two key enzymes in the oxidative and non-oxidative phase of PPP and play a role in nucleotide bio-synthesis $(31,32)$. In Western blots, we observed that aspirin acetylated both G6PD (Fig. 5A) and

transketolase (Fig. 5C), where as in the untreated control, acetylation of these enzymes were undetected. The cell lysates used for immunoprecipitation contained similar amounts of G6PD and transketolase protein levels suggesting that aspirin does not alter their expression levels (Fig. 5B and D). Similar results were obtained in HT-29 cells (data not shown).

Acetylation of G6PD is associated with decreased enzyme activity. To determine if acetylation of G6PD affects its enzyme activity, HCT-116 cells were untreated or treated with different concentrations of aspirin (100-500 $\mu \mathrm{M})$, G6PD activity was measured. Fig. 6 demonstrates that aspirin progressively inhibited G6PD activity particularly beginning at $200 \mu \mathrm{M}$. Numerically, the percentage inhibition was found to be $7 \%$ at $100 \mu \mathrm{M}, 17 \%$ at $200 \mu \mathrm{M}, 20 \%$ at $300 \mu \mathrm{M}, 23 \%$ at $400 \mu \mathrm{M}$ and $35 \%$ at $500 \mu \mathrm{M}$. Similarly, treatment of HT-29 cells with aspirin also reduced G6PD activity in (data not shown). This suggests that acetylation of G6PD by aspirin causes a decrease in its enzyme activity.

\section{Discussion}

Aspirin's chemopreventive effects are increasingly being recognized; however, a plausible explanation for its anti-cancer effect is only beginning to be understood. We previously demonstrated in the human breast cancer cell line MDA-MB-231 that aspirin acetylates the tumor suppressor protein p53 at K382, and induces expression of its target genes (17). The present study was carried out to explore if aspirin acetylates other cellular proteins and modulate their function, which potentially could explain its anti-cancer effects. In this study, we demonstrate for the first time that aspirin, within the range of physiologically achievable concentration (100-300 $\mu \mathrm{M})(33-35)$, acetylated multiple proteins at lysine residues in both HCT-116 and HT-29 colon cancer cells. Through mass spectrometry and immunoblotting, we identified several acetylation targets for aspirin. These include metabolic enzymes, cytoskeletal proteins, heat shock proteins, histones as well as some mitochondrial proteins. This is an important observation because, till date, very few proteins are known to be acetylated in cells exposed to aspirin $(8,17,36)$. Thus, our finding extends the previously known list of proteins acetylated by aspirin suggesting that some of its unexplained therapeutic properties may occur through acetylation of proteins.

In this study, we identified a total of 49 proteins as potential targets for aspirin mediated acetylation using mass-spectrometry. Among these, few proteins that are identified may not be the true targets of aspirin, as unmodified proteins may co-precipitate with acetylated proteins by non-specific adsorption in immunoprecipitation reactions. Among the proteins that were identified as potential targets, we focused on the enzymes of glucose metabolism due to their importance in cancer (24). Rapidly growing cancer cells are known to have glycolytic rates that are up to 200 times higher than that of normal cells (23). This increased rate of glucose utilization has been attributed to mainly to the expression of PKM2 isoform in cancer cells (28), although some studies have implicated a role for hexokinase II in this process (37). Under normal conditions PKM2 is expressed in embryonic and rapidly growing cells; however, its expression is observed at elevated levels in cancer cells (24). Similarly, another report showed that LDH-A is important for tumor progression (29). In our study, six enzymes in the glycolytic pathway were 


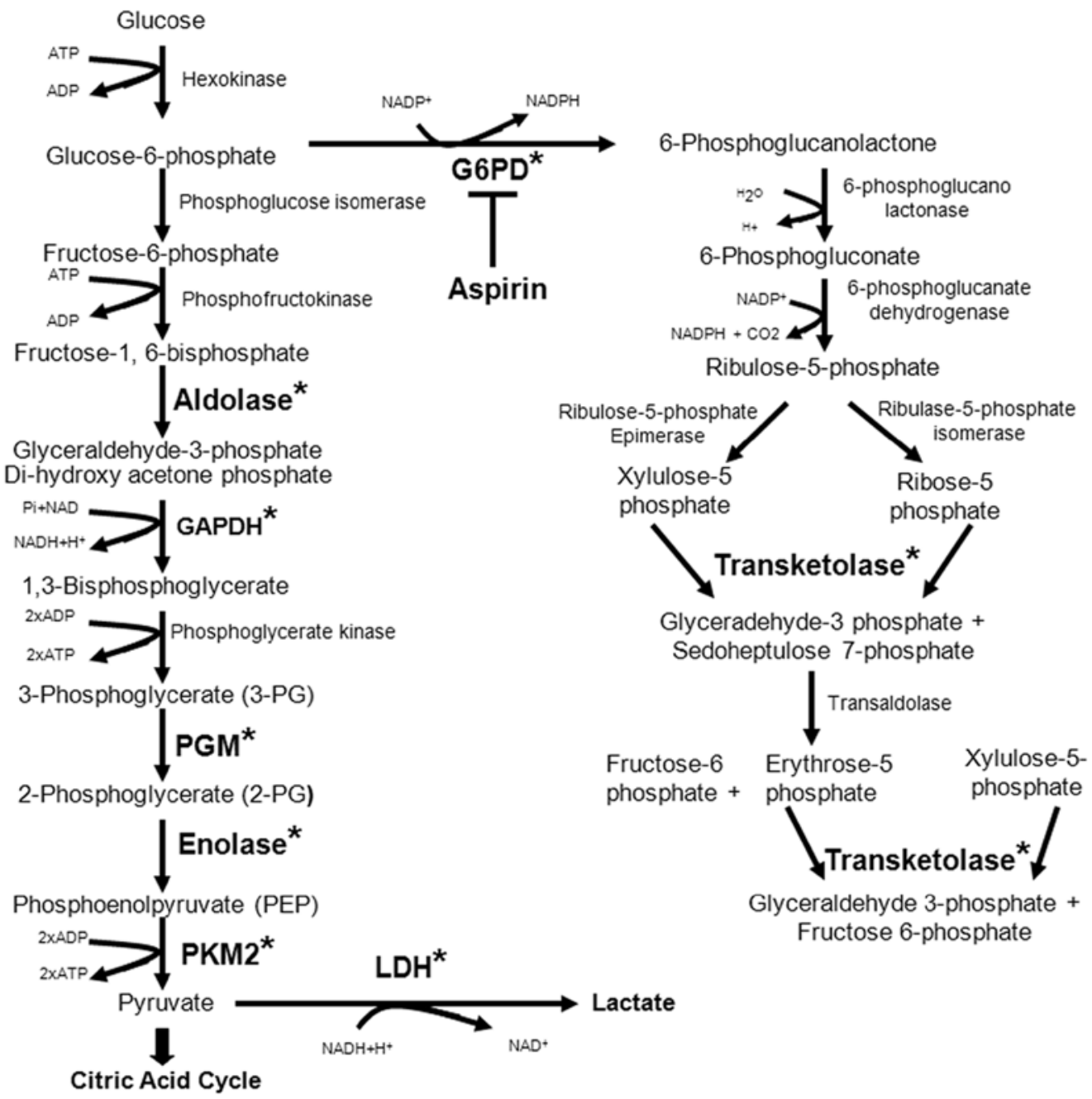

Figure 7. Model depicting the acetylation targets of aspirin on glycolysis/pentose phosphate pathway enzymes. Aspirin acetylates six enzymes in glycolysis and two enzymes in pentose phosphate pathway (shown in bold and with an asterisk). Note that enzyme inhibition is observed with G6PD.

found to be acetylated upon aspirin treatment. These include aldolase, GAPDH, PGM, enolase, PKM2, LDH-A and LDH-B. Among these, most abundant acetylation was observed for enolase by aspirin. Interestingly, enolase was constitutively acetylated although to a lower level and this is consistent with previous reports (38). Despite acetylation of PKM2, its activity remained unchanged upon aspirin treatment. Aspirin also did not alter PKM2 phosphorylation at $\mathrm{Y}^{105}$ which further supports the view that enzyme activity remains unaltered following aspirin treatment. Some reports show that PKM2 is differentially compartmentalized within the cells (24); however, it is not clear if aspirin-induced acetylation affects its compartmentalization. Similarly, LDH activity was also unaffected upon aspirin treatment. In immunoblotting experiments we did not see acetylation of hexokinase II by aspirin (data not shown) which suggests that some enzymes in glycolytic pathway are not the targets of aspirin. Our results suggest that aspirin post-translationally modifies a wide range of proteins with probably neutral impact on bulk protein function.

While the amount of protein concentrated in the immunoprecipitation reaction was low and posed some limits on detection by mass spectrometry, we identified G6PD and transketolase as the target of aspirin mediated acetylation by using immunobloting experiments. This observation reflects the well-known fact that immunoblotting techniques are more sensitive detection methods than mass spectrometry, particularly for low abundance proteins. G6PD is the key regulatory enzyme in PPP and necessary for the synthesis of nucleotides and nucleic acids (39-41). Recent studies have demonstrated that G6PD activity is suppressed in cells containing wild-type p53 through a direct inhibition; whereas, it is not subjected to such negative regulation in cells containing mutant p53 (42). This has been implicated in the increased consumption of glucose in tumor cells containing mutant $\mathrm{p} 53$. Our previous reports show that aspirin has the ability to acetylate and activate both wild-type and mutant p53 (17). In this study, we observed that acetylation of G6PD by aspirin was associated with inhibition of its activity. This is a novel finding because the targeted inhibition of G6PD has been proposed as a strategy to inhibit nucleic acid synthesis in cancer therapy (43). Several studies have demonstrated the potential of inhibiting G6PD to reduce cell proliferation in vitro and in vivo $(31,44,45)$. For example, the ability of the dehydroepiandrosterone to inhibit G6PD and to inhibit cancer cell proliferation has been documented $(32,40,44)$. It is not clear at this stage if G6PD inhibition 
observed in aspirin treated cells is due to an interaction between acetylated p53 and G6PD, or is the result of acetylation of G6PD itself. Our finding that aspirin inhibits G6PD suggests that inhibition of PPP could be one mechanism that contributes to the anti-cancer/anti-proliferative effects of aspirin.

In this study, we have not investigated the importance of the increased acetylation of other proteins that were identified through mass-spectrometry for their significance in aspirin's anti-cancer effects. These include, heat shock proteins, histones, ribosomal proteins and annexins whose modification could also play a role. For example, hyper-acetylation of HSP-90 $\alpha$ has been shown to increase its association with MMP-2 leading to increased tumor cell invasiveness (46). One of the proteins that was identified with significant Mascot score in mass-spectrometry is HSP90 $\alpha$. We have confirmed its acetylation in immunoblotting experiments (data not shown). Annexin, a cytosketal protein, is involved in diverse cellular processes such as epithelial cell motility and cell matrix interactions (47). It has been shown that histone acetylation has the capacity to destabilize the chromatin polymer through charge neutralization of the basic lysine residues leading to changes in the chromatin structure and transcription of genes (30). Moreover, inhibitors of histone deacetylases have been used in chemotherapy (48). Chemical acetylation by aspirin can play a role in shifting the equilibrium of the enzymatic acetylation/deacetylation process. It remains to be determined how acetylation of these proteins could affect their functions and whether these contribute to aspirin's anti-cancer effects.

Protein lysine acetylation over the past decade has emerged as a key post-translational modification in cellular regulation of both histone and transcription factors (49). Importantly, work carried out in the last two years show that acetylation of proteins is a wide spread phenomenon occurring in a variety of proteins encompassing proteins involved in metabolic pathways, RNA splicing, cell cycle, DNA repair, nuclear proteins, cytoskeleton and nuclear transport (19). One recent study demonstrated in human liver cells (21) that every enzyme involved in glycolysis, fatty acid and glycogen metabolism, tricarboxylic and urea cycles are acetylated. Changes in acetylation status affect the enzymatic activity to allow the cells to respond changes in the metabolic demands $(20,21)$. Thus, modification of proteins through acetylation has been recognized to be as important as modification through phosphorylation $(50,51)$. The finding that aspirin can acetylate multiple proteins suggests that it may exert its chemopreventive effects through alteration of the functional activity of few selective proteins. This represents an important area of future studies.

\section{Acknowledgements}

This project was supported by a grant from National Institute of Health (5R03CA133061-02) to G.J.B. We thank Dr Kalkunte Srivenugopal and Dr Vardan Karamyan for advice and useful discussions. We also thank Kevin Welle and the University of Rochester Proteomics Center for mass spectrometry analysis.

\section{References}

1. Flossmann E and Rothwell PM: Effect of aspirin on long-term risk of colorectal cancer: consistent evidence from randomised and observational studies. Lancet 369: 1603-1613, 2007.

2. Chan AT and Giovannucci EL: Primary prevention of colorectal cancer. Gastroenterology 138: 2029-2043, 2010.
3. Harris RE, Beebe-Donk J, Doss H and Burr Doss D: Aspirin, ibuprofen, and other non-steroidal anti-inflammatory drugs in cancer prevention: a critical review of non-selective COX-2 blockade (review). Oncol Rep 13: 559-583, 2005.

4. Luciani MG, Campregher C and Gasche C: Aspirin blocks proliferation in colon cells by inducing a G1 arrest and apoptosis through activation of the checkpoint kinase ATM. Carcinogenesis 28: 2207-2217, 2007.

5. Hanif R, Pittas A, Feng Y, et al: Effects of nonsteroidal antiinflammatory drugs on proliferation and on induction of apoptosis in colon cancer cells by a prostaglandin-independent pathway. Biochem Pharmacol 52: 237-245, 1996.

6. Chan AT, Ogino S and Fuchs CS: Aspirin use and survival after diagnosis of colorectal cancer. JAMA 302: 649-658, 2009.

7. Kopp E and Ghosh S: Inhibition of NF-kappa B by sodium salicylate and aspirin. Science 265: 956-959, 1994.

8. Roth GJ, Machuga ET and Ozols J: Isolation and covalent structure of the aspirin-modified, active-site region of prostaglandin synthetase. Biochemistry 22: 4672-4675, 1983.

9. Vane JR and Botting RM: The mechanism of action of aspirin. Thromb Res 110: 255-258, 2003.

10. Oshima M, Dinchuk JE, Kargman SL, et al: Suppression of intestinal polyposis in Apc delta716 knockout mice by inhibition of cyclooxygenase 2 (COX-2). Cell 87: 803-809, 1996.

11. Soh JW and Weinstein IB: Role of COX-independent targets of NSAIDs and related compounds in cancer prevention and treatment. Prog Exp Tumor Res 37: 261-285, 2003.

12. Yin MJ, Yamamoto Y and Gaynor RB: The anti-inflammatory agents aspirin and salicylate inhibit the activity of $\mathrm{I}(\mathrm{kappa}) \mathrm{B}$ kinase-beta. Nature 396: 77-80, 1998.

13. Schwenger P, Bellosta P, Vietor I, Basilico C, Skolnik EY and Vilcek J: Sodium salicylate induces apoptosis via p38 mitogen-activated protein kinase but inhibits tumor necrosis factor-induced c-Jun N-terminal kinase/stress-activated protein kinase activation. Proc Natl Acad Sci USA 94: 2869-2873, 1997.

14. Martinez ME, O'Brien TG, Fultz KE, et al: Pronounced reduction in adenoma recurrence associated with aspirin use and a polymorphism in the ornithine decarboxylase gene. Proc Natl Acad Sci USA 100: 7859-7864, 2003.

15. Caterson RJ, Duggin GG, Horvath J, Mohandas J and Tiller D: Aspirin, protein transacetylation and inhibition of prostaglandin synthetase in the kidney. Br J Pharmacol 64: 353-358, 1978.

16. Green FA and Jung CY: Acetylation of erythrocytic membrane peptides by aspirin. Transfusion 21: 55-58, 1981.

17. Alfonso LF, Srivenugopal KS, Arumugam TV, Abbruscato TJ, Weidanz JA and Bhat GJ: Aspirin inhibits camptothecin-induced p $21^{\mathrm{CIP1}}$ levels and potentiates apoptosis in human breast cancer cells. Int J Oncol 34: 597-608, 2009.

18. Alfonso LF, Srivenugopal KS and Bhat GJ: Does aspirin acetylate multiple cellular proteins? (Review). Mol Med Rep 2: 533-537, 2009.

19. Choudhary C, Kumar C, Gnad F, et al: Lysine acetylation targets protein complexes and co-regulates major cellular functions. Science 325: 834-840, 2009.

20. Wang Q, Zhang Y, Yang C, et al: Acetylation of metabolic enzymes coordinates carbon source utilization and metabolic flux. Science 327: 1004-1007, 2010.

21. Zhao S, Xu W, Jiang W, et al: Regulation of cellular metabolism by protein lysine acetylation. Science 327: 1000-1004, 2010.

22. Pinckard RN, Hawkins D and Farr RS: In vitro acetylation of plasma proteins, enzymes and DNA by aspirin. Nature 219: 68-69, 1968.

23. Warburg O: On the origin of cancer cells. Science 123: 309-314, 1956.

24. Mazurek S: Pyruvate kinase type M2: A key regulator of the metabolic budget system in tumor cells. Int J Biochem Cell Biol (In press).

25. Cairns RA, Harris IS and Mak TW: Regulation of cancer cell metabolism. Nat Rev Cancer 11: 85-95, 2011.

26. Roberts MS, Rumble RH, Wanwimolruk S, Thomas D and Brooks PM: Pharmacokinetics of aspirin and salicylate in elderly subjects and in patients with alcoholic liver disease. Eur J Clin Pharmacol 25: 253-261, 1983.

27. Hitosugi T, Kang S, Vander Heiden MG, et al: Tyrosine phosphorylation inhibits PKM2 to promote the Warburg effect and tumor growth. Sci Signal 2: ra73, 2009.

28. Christofk HR, Vander Heiden MG, Harris MH, et al: The M2 splice isoform of pyruvate kinase is important for cancer metabolism and tumour growth. Nature 452: 230-233, 2008.

29. Le A, Cooper CR, Gouw AM, et al: Inhibition of lactate dehydrogenase A induces oxidative stress and inhibits tumor progression. Proc Natl Acad Sci USA 107: 2037-2042, 2010. 
30. Basu A, Rose KL, Zhang J, et al: Proteome-wide prediction of acetylation substrates. Proc Natl Acad Sci USA 106: 13785-13790, 2009.

31. Ramos-Montoya A, Lee WN, Bassilian S, et al: Pentose phosphate cycle oxidative and nonoxidative balance: A new vulnerable target for overcoming drug resistance in cancer. Int J Cancer 119: 2733-2741, 2006.

32. Boros LG, Lee PW, Brandes JL, et al: Nonoxidative pentose phosphate pathways and their direct role in ribose synthesis in tumors: is cancer a disease of cellular glucose metabolism? Med Hypotheses 50: 55-59, 1998.

33. Rowland M, Riegelman S, Harris PA and Sholkoff SD: Absorption kinetics of aspirin in man following oral administration of an aqueous solution. J Pharm Sci 61: 379-385, 1972.

34. Liyasova MS, Schopfer LM and Lockridge O: Reaction of human albumin with aspirin in vitro: mass spectrometric identification of acetylated lysines 199, 402, 519, and 545. Biochem Pharmacol 79: 784-791, 2010.

35. Needs CJ and Brooks PM: Clinical pharmacokinetics of the salicylates. Clin Pharmacokinet 10: 164-177, 1985.

36. Cherian M and Abraham EC: Glycation of human lens crystallins: effect of age and aspirin treatment. Ophthalmic Res 25: 349-354, 1993.

37. Mathupala SP, Ko YH and Pedersen PL: Hexokinase-2 bound to mitochondria: cancer's stygian link to the 'Warburg effect' and a pivotal target for effective therapy. Semin Cancer Biol 19: 17-24, 2009.

38. Zhou W, Capello M, Fredolini C, et al: Mass spectrometry analysis of the post-translational modifications of alpha-enolase from pancreatic ductal adenocarcinoma cells. J Proteome Res 9: 2929-2936, 2010.

39. Vizan P, Alcarraz-Vizan G, Diaz-Moralli S, Solovjeva ON, Frederiks WM and Cascante M: Modulation of pentose phosphate pathway during cell cycle progression in human colon adenocarcinoma cell line HT29. Int J Cancer 124: 2789-2796, 2009.

40. Tian WN, Braunstein LD, Pang J, et al: Importance of glucose6-phosphate dehydrogenase activity for cell growth. J Biol Chem 273: 10609-10617, 1998.
41. Board M, Humm S and Newsholme EA: Maximum activities of key enzymes of glycolysis, glutaminolysis, pentose phosphate pathway and tricarboxylic acid cycle in normal, neoplastic and suppressed cells. Biochem J 265: 503-509, 1990.

42. Jiang P, Du W, Wang X, et al: p53 regulates biosynthesis through direct inactivation of glucose-6-phosphate dehydrogenase. Nat Cell Biol 13: 310-316, 2011.

43. Tennant DA, Duran RV and Gottlieb E: Targeting metabolic transformation for cancer therapy. Nat Rev Cancer 10: 267-277, 2010.

44. Boros LG, Puigjaner J, Cascante M, et al: Oxythiamine and dehydroepiandrosterone inhibit the nonoxidative synthesis of ribose and tumor cell proliferation. Cancer Res 57: 4242-4248, 1997.

45. Rais B, Comin B, Puigjaner J, et al: Oxythiamine and dehydroepiandrosterone induce a G1 phase cycle arrest in Ehrlich's tumor cells through inhibition of the pentose cycle. FEBS Lett 456: 113-118, 1999.

46. Yang Y, Rao R, Shen J, et al: Role of acetylation and extracellular location of heat shock protein 90alpha in tumor cell invasion. Cancer Res 68: 4833-4842, 2008.

47. Guzman-Aranguez A, Olmo N, Turnay J, et al: Differentiation of human colon adenocarcinoma cells alters the expression and intracellular localization of annexins A1, A2, and A5. J Cell Biochem 94: 178-193, 2005.

48. Lane AA and Chabner BA: Histone deacetylase inhibitors in cancer therapy. J Clin Oncol 27: 5459-5468, 2009.

49. Spange S, Wagner T, Heinzel T and Kramer OH: Acetylation of non-histone proteins modulates cellular signalling at multiple levels. Int J Biochem Cell Biol 41: 185-198, 2009.

50. Kouzarides T: Acetylation: a regulatory modification to rival phosphorylation? EMBO J 19: 1176-1179, 2000.

51. Norvell A and McMahon SB: Cell biology. Rise of the rival. Science 327: 964-965, 2010. 